\title{
A GaN-based DC/DC converter for e-vehicles applications
}

\author{
Eduardo F. de Oliveira ${ }^{1}$, Sebastian Sprunck ${ }^{2}$, Jonas Pfeiffer ${ }^{2}$ (D) and Peter Zacharias ${ }^{2}$ \\ ${ }^{1}$ HUAWEI TECHNOLOGIES \\ Nuremberg Research Center \\ Südwestpark 48 \\ 90449 Nürnberg, Germany \\ Tel.: $+49 /(0) 1590-4450835$ \\ Fax: +49 / (0)911 - 255223090 \\ E-Mail: eduardof.oliveira@huawei.com \\ URL: http://www.huawei.com \\ ${ }^{2}$ UNIVERSITY OF KASSEL \\ KDEE-EVS \\ Wilhelmshöher Allee 71 \\ 34121 Kassel, Germany \\ Tel.: +49 / (0)561 - 8046344 \\ Fax: +49 / (0)561 - 8046521 \\ E-Mail: peter.zacharias@uni-kassel.de \\ URL: http://www.uni-kassel.de/eecs/evs
}

\section{Acknowledgements}

The authors acknowledge the financial support by the German Federal Ministry of Education and Research (BMBF) in the framework of project HELENE number 16EMO0234. Responsibility for the contents of this publications lies with the authors.

\section{Keywords}

«Gallium Nitride (GaN)», «Magnetic device», «Power converters for EV», «Thermal design», «Wide bandgap devices»

\begin{abstract}
This paper presents a $4 \mathrm{~kW}$ GaN-based isolated bidirectional DC/DC converter suitable for on-board auxiliary power supply systems, interconnecting the HV batteries with the LV on-board power supply system. To enable the trend towards higher levels of electrification, a second LV level of $48 \mathrm{~V}$ is introduced to supply the high-power consumers, relieving the $12 \mathrm{~V}$ grid on the one hand and/or avoiding using additional costly isolated converters connected to the HV batteries on the other. The built converter prototype aims to demonstrate higher efficiency, compactness and lower filter requirements of the proposed circuit.
\end{abstract}

\section{Introduction}

In the course of the debate on the reduction of fossil fuel emissions, electromobility became an important topic in politics and society. In conjunction with the expansion of renewable energies, the electrification of transport can be a useful approach. Especially in urban areas, electric vehicles can be a solution to reduce fossil fuel emissions drastically. The development of electric vehicles has increased substantially in recent years. However, automotive manufactures still have to deal with numerous challenges, especially relating to range extension and load management. Wide band gap semiconductors have the potential to improve the efficiency of the used power electronic devices and can therefore be a part of the required solutions.

The common structure of a BEV is composed by two on-board power systems. The HV power system comprises the traction batteries with voltage levels up to $500 \mathrm{~V}$ and is responsible for feeding the drive train of the vehicle. In turn, the LV power system, with a rated voltage of $12 \mathrm{~V}$, feeds most of the electric consumers. Its power demand easily reaches around $3 \mathrm{~kW}$ in contemporary vehicles [1]. The increase of the on-board power with the addition of new electric components motivates the introduction of a third voltage level of $48 \mathrm{~V}$, which enables the supply of high-power consumers, especially in high-end model cars [2], [3]. Compared to the $12 \mathrm{~V}$ battery, the $48 \mathrm{~V}$ network can provide 4 times more power at the same current level, while remaining below the automotive HV 


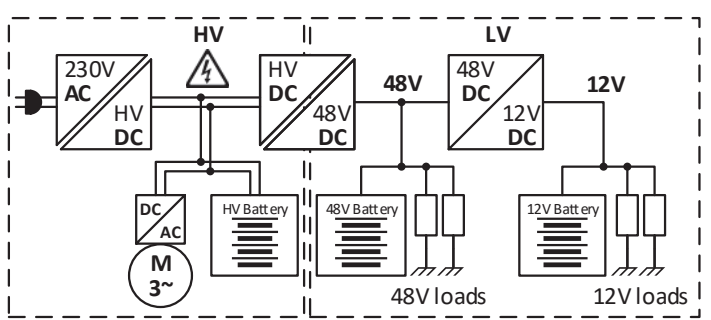

Fig. 1: State-of-the-art on-board power supply system block diagram [1] threshold of $60 \mathrm{~V}$, where the standards require the use of costly shielding, conduits and connectors that would increase the cost rapidly [3]. The $48 \mathrm{~V}$ system reliefs the $12 \mathrm{~V}$ grid on the one hand and/or avoids using additional costly isolated converters connected to the HV batteries on the other [2][4]. Moreover, $12 \mathrm{~V}$ and $48 \mathrm{~V}$ electrical grids can co-exist for the foreseeable future. A joint effort from GM, HELLA and COSMX has developed a way to provide both voltage levels from a single system [5]. Called MODACS (Multiple Output Dynamically Adjustable Capacity System), the invention eliminates not only the need for separate $12 \mathrm{~V}$ and $48 \mathrm{~V}$ batteries, but also the losses coming from the DC/DC converter between both grids [6].

In turn, the connection between the $48 \mathrm{~V}$ supply and the HV battery must be executed by using a galvanically isolated DC/DC converter. Using a highly dynamic converter would allow the reduction or even the elimination of the $48 \mathrm{~V}$ battery. The converter proposed in this paper works as an on-board power supply system connecting the HV side to the $48 \mathrm{~V}$ batteries. The focus of this paper lies on the optimal electrical implementation of gallium nitride semiconductors and the inherent challenge of removing its dissipated heat. Further focuses are the thermal management of the magnetics in combination with their arrangement to achieve a high power density. By iterative optimization, a setup with minimum losses and volume is identified, constructed and validated experimentally.

\section{Investigated topology}

One of the most attractive soft-switching isolated DC-DC converter topologies for high power applications, where bidirectional power flow, robust operation and step-up/step-down functionality are required, is the three-phase DAB presented in [7]. In recent years, some new topologies based on the $3 \mathrm{ph}-\mathrm{DAB}$ have been developed, for instance the Y-YY 3ph-DAB proposed in [8]. A second low voltage bridge is added to the original circuit, so that the topology now works with six transformers, each phase having two transformers. This configuration presents two transformers per phase connected in series on the high voltage side and paralleled three-phase bridges on the low voltage side. The new turn ratio of each transformer is now divided by two. Therefore, the voltage across the transformer's HV winding as well as the currents through the transformer's LV winding and through the LV switches are also divided by two. A similar approach is proposed in [1]. However, in contrast to the previous circuit, one of the secondaries presents a delta connection, originating the wye wyedelta (Y-Y $\Delta$ ) 3ph-DAB (see Fig. 2). The Y-Y $\Delta$ connection results in a 12-pulse converter, reducing therefore the output filter requirements due to the higher frequency seen by the output filter. Other than for the conventional 3ph-DAB, power flow is controlled by applying a phase-shift angle of $\Phi$ and

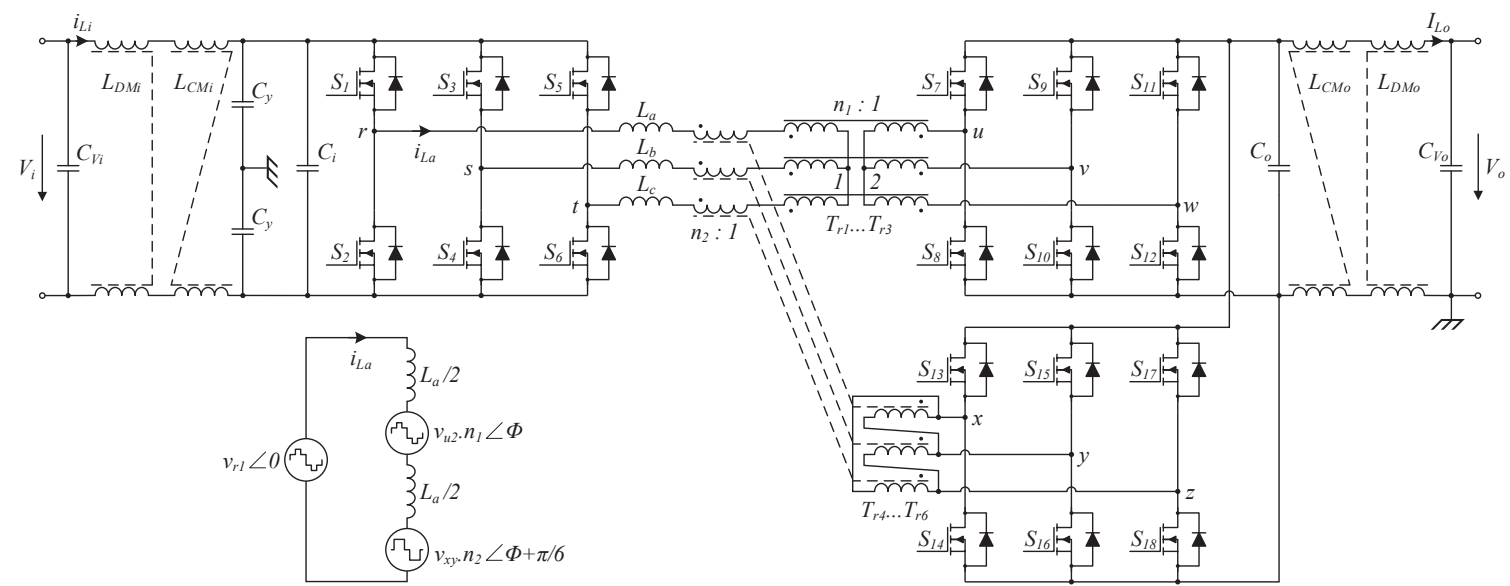

Fig. 2: Simplified topology of the 12-pulse Y-Y $\Delta 3$ ph-DAB 
$\Phi+\pi / 6$ between the gating signals of the input and the $\mathrm{Y}$ and $\Delta$ output bridges, respectively. After an extensive topology benchmark, the Y-Y $\Delta 3$ ph-DAB is chosen as the more advantageous alternative regarding losses, volume and output filter efforts for this application. A detailed description of the operation modes and an extensive steady-state analysis of the $\mathrm{Y}-\mathrm{Y} \Delta 3 \mathrm{ph}-\mathrm{DAB}$ can be found in [1].

\section{Integrated Magnetics}

The storage chokes are essential for the operation of DAB converters. They can be designed as discrete magnetic components or integrated within the transformer's leakage inductance. Regarding the integrated approach, three different setups were investigated. In the first design, primary and secondary windings are wound on the entire length of the center leg of the used PQ-core (helical windings). The leakage inductance is adjusted by varying the horizontal distance between both windings using spacers. Due to the high coupling factor between the coils, the achievable value of the leakage inductance is quite limited. In the second design, the primary winding is wound on the upper part of the center leg, whereas the secondary winding is placed on the lower part (disc windings). Vertical spacers or winding chambers are used to adjust the required leakage inductance. In comparison to the first method, a higher leakage inductance can be reached, whilst degrading the efficiency. The third design extends the second one by introducing ferrite plates between the coils. The ferrite plates concentrate the flux leakage, which results in a higher leakage inductance. However, the design and construction processes of the magnetic device become more difficult, especially if a concrete leakage inductance value is required. Furthermore, it is important to prevent a saturation of the ferrite plates what would lead to a significant drop of the transformer's flux leakage. For these reasons, standard disc windings are used in the final converter. Due to geometric constrains, the delivered transformers do by far not reach the desired leakage inductance of $10.8 \mu \mathrm{H}$ (being for each transformer the half), presenting an average total value of ca. $7.8 \mu \mathrm{H}$. Nonetheless, no additional inductors are added to the final prototype.

\section{Characterization of GaN E-HEMT switching losses}

The characterization of GaN E-HEMTs is performed using a standard double pulse test (DPT). The test environment has to be improved regarding its $d v / d t$ and $d i / d t$ immunity, which is realized using suitable common mode filters on every external connection. The measured switching losses, though subject to uncertainties stemming from the used current shunt [9], are used as a benchmark for several gate driver and switching cell setups. Using an automated measurement setup, the GaN semiconductor's switching losses are examined regarding their dependency on DC voltage, switching current, gate turn-on and turn-off voltages, gate resistances, dead time and junction temperature. These investigations are repeated for several combinations of different gate drivers and gate networks, resulting in a broad database for application-specific loss minimization. An excerpt of the measurements' results at room temperature is displayed in Fig. 3.

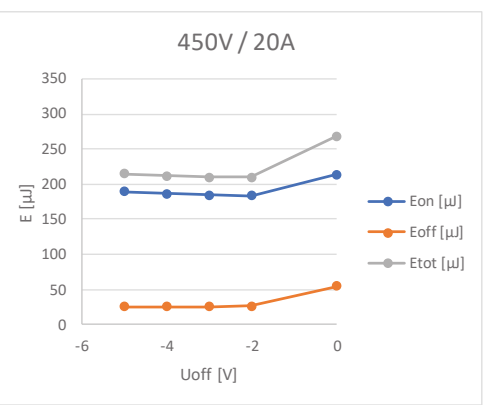

a)

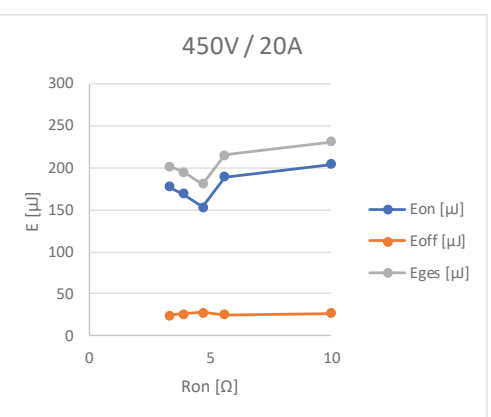

b)

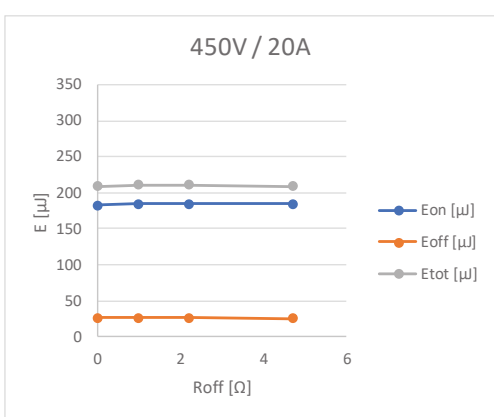

c)

Fig. 3: Influence of different setup parameters on the measured switching energies. a) $E_{S W}$ vs. $U_{\text {off }}$ for $R_{o n}=5.6 \Omega, R_{\text {off }}=0 \Omega$ and $U_{\text {on }}=5 \mathrm{~V}$. b) $E_{s w}$ vs. $R_{\text {on }}$ for $R_{\text {off }}=0 \Omega, U_{\text {on }}=5 \mathrm{~V}$ and $U_{\text {off }}=$ $-2 \mathrm{~V}$. c) $E_{s w}$ vs. $R_{\text {off }}$ for $R_{o n}=10 \Omega, U_{o n}=6 \mathrm{~V}$ and $U_{o f f}=-2 \mathrm{~V}$ 


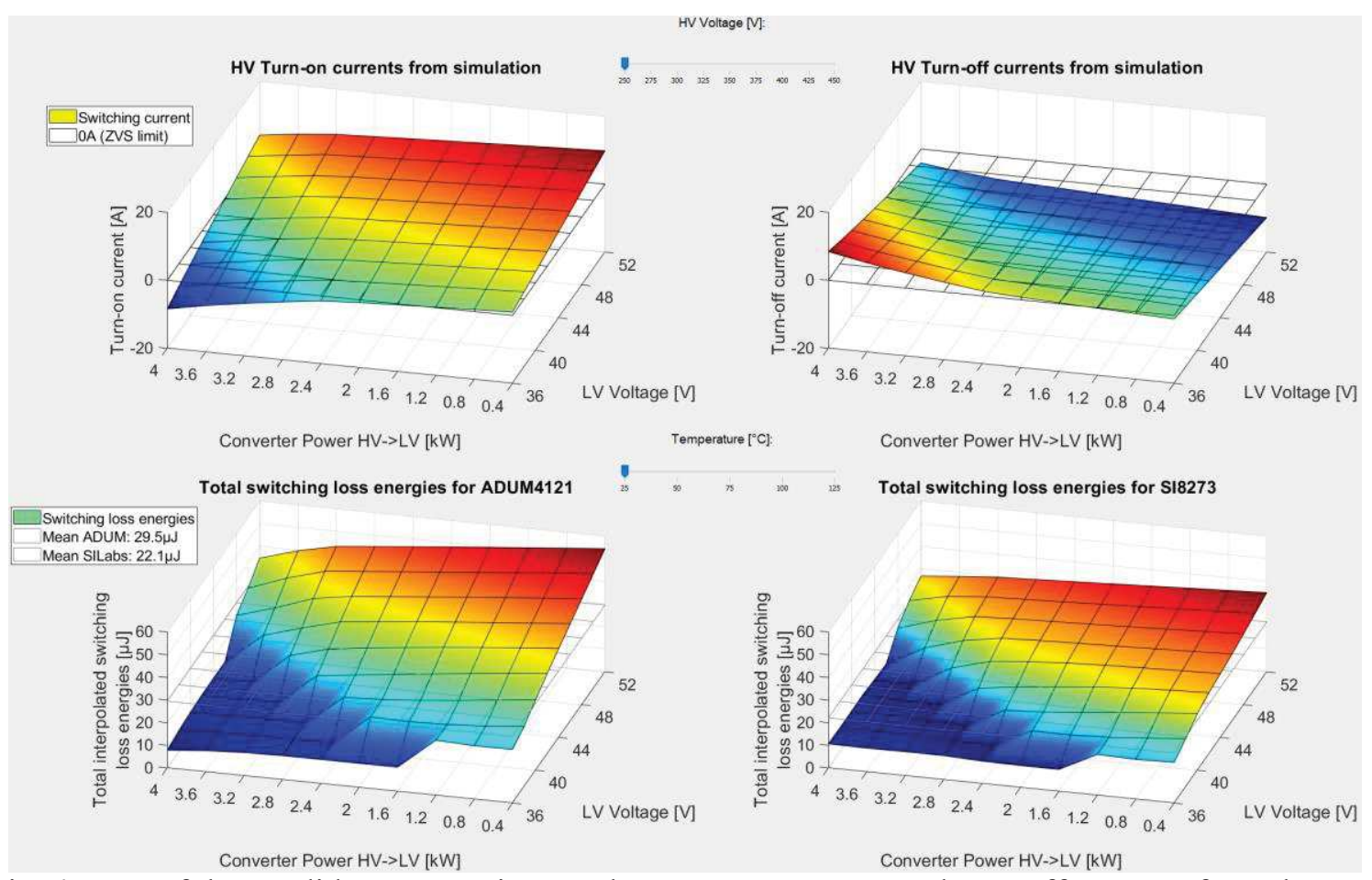

Fig. 4: GUI of the candidate comparison tool. Top row: Turn-on and turn-off currents from the simulation according to the selected operation conditions. Bottom row: Switching loss results for two different drivers. The HV voltage and chip temperature can be adjusted using the sliders

It is evident that the turn-off losses are an order of magnitude below the turn-on losses and can hardly be influenced at all, save for turn-off voltages between $0 \mathrm{~V}$ and $-2 \mathrm{~V}$. Turn-on losses, on the other hand, dominate the overall switching losses and can be influenced by the gate resistor network as well as the turn-on and turn-off voltages. The latter effect is rather surprising and was therefore investigated more closely in [10]. The data recorded by this test bench are then integrated into an evaluation tool that uses the simulated operation points of the selected topology to calculate the total semiconductor switching losses. These losses are calculated for the most promising driver and setup candidates and consecutively compared regarding certain criteria, such as operating voltage and chip temperature. An excerpt of the tool's GUI is shown in Fig. 4.

\section{Physical setup and thermal design}

Although GaN can operate at very high junction temperatures, it still needs to be packaged in a case. This case limits the maximum allowable operating temperature, making an effective cooling system a necessity. Liquid cooling systems, present in most cars, can be used for this purpose but it has to be ensured that the cooling terminal of the $\mathrm{GaN}$ semiconductor package is thermally well connected to the heat sink. The required creepage distance for the HV switches as well as the amount of LV switches pose challenges regarding the required PCB and cooler surface areas. If the switching cells were arranged side-by-side, a very large area would be required, adding a lot of volume through the vertical size of the transformers alone and thus reducing the achievable power density. Through iterative testing, a 3D setup is developed to minimize the converter's volume. This allows the transformers, switches as well as filters to be connected closely to the cooling channel, while keeping the base area of the converter in an acceptable range (see Fig. 5).

The top-side cooled GaN semiconductors are mounted on the bottom of the PCB. Their substrates are internally connected to the thermal pads on top, which in turn are coupled to the heatsink by means of a flexible thermal interface material (TIM). To avoid device failure due to thermomechanical bending stress, the heat sink is designed with cavities to fit the semiconductors and the TIMs, as shown in Fig. 6a. The PCB area opposite to the switches is lightly pressed against the heatsink using 3D printed 

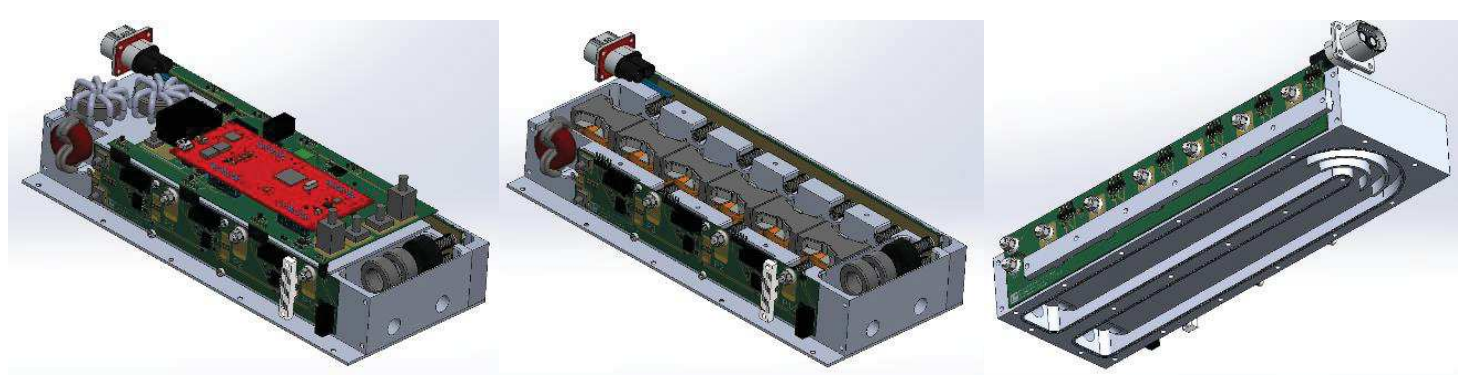

Fig. 5: CAD model of the 3D-PCB-concept and the cooling system (casing not shown)

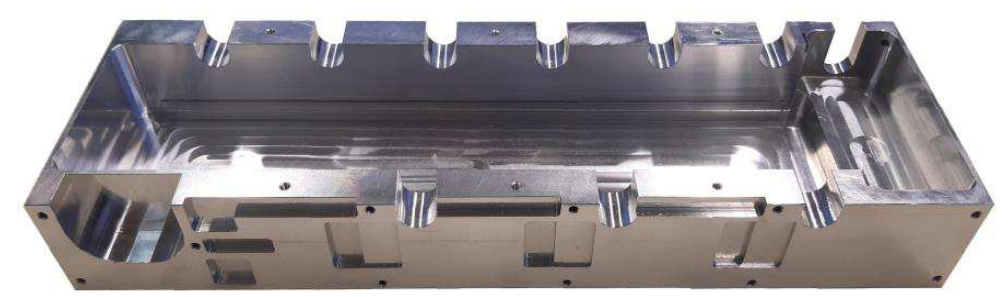

a)

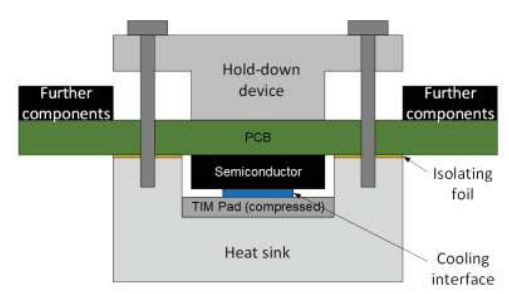

b)

Fig. 6: a) Details of the cavities on the water cooling heatsink and b) schematic cross-section view of a GaN semiconductor mounted in a cavity (not to scale)

hold-down devices (Fig. 6b) to ensure constant thermal contact between semiconductors, TIMs and the heat sink. In this manner, the GaN devices are protected from excessive bending stresses due to the screw mounting and thus are almost solely subjected to thermal expansion [11]. This approach provides a compact and thermally stable connection, but great care has to be taken regarding device tolerances, TIM compression and manufacturing precision, especially when using very thin TIMs.

As the transformers have to be placed in-between the HV and LV switching cells, they are embedded into a cavity below the control and measurement PCB which is placed on top. This board is protected against the transformers' shifting magnetic fields by inserting a grounded metal sheet between them, acting as an EMI shield. As the LV filters are subjected to high currents, they too are embedded into the cavity for cooling purposes. The HV filters on the other hand are loaded with lower currents, requiring no tight coupling to the cooling system. As the cooling fluid should pass all the semiconductors and the transformers to avoid overheating, a $180^{\circ}$ turn of the flow was designed into the channel. Several simulative iterations were performed, accounting for chip orientation, cooling channel design and chip heating. With this approach, an optimal overall setup could be found, allowing a total volume of $298 \times 127 \times 77 \mathrm{~mm}(\approx 2.9$ liters), resulting in a power density of approx. $1.38 \mathrm{~kW} / \mathrm{L}$.

\section{Optimization and 3ph-DAB circuits' benchmark}

Reconfiguring the transformer's arrangement, the implemented prototypes are not only able to operate as a 12-pulse Y-Y $\triangle 3$ ph-DAB, but also as both conventional 3ph-DAB [7] and Y-YY 3ph-DAB [8]. An optimization procedure, based on comprehensive analytical models of these three topologies, has been developed and leads to the optimal parameters to achieve the best compromise between compactness and efficiency. The best possible combination between losses, volume and required dc-link capacitance can be found after millions of iterations (see Fig. 7).

Comparing the best obtained designs of the 12-pulse Y-Y $\Delta$ 3ph-DAB and of the conventional 3ph$\mathrm{DAB}$, the calculations indicate a reduction of approximately $22 \%, 9 \%$ and $70 \%$ concerning overall losses, transformer volume and required output capacitance, respectively. In turn, comparing the 12pulse Y-Y $\triangle 3$ ph-DAB to the Y-YY 3-phase DAB, the total transformer volume remains the same for both, however the overall losses and the output capacitance are reduced by $6 \%$ and $70 \%$, respectively. Besides exhibiting lower average total losses and lower total magnetic core volume, the biggest advantage of the Y-Y $\Delta 3$ ph-DAB compared to the conventional 3ph-DAB and to the Y-YY 3 ph-DAB is the much lower current ripple at the secondary side, thereby lower VA rating of filter capacitors owing to the interleaving effect of the $\mathrm{Y}-\mathrm{Y} \Delta$ connection [1]. 


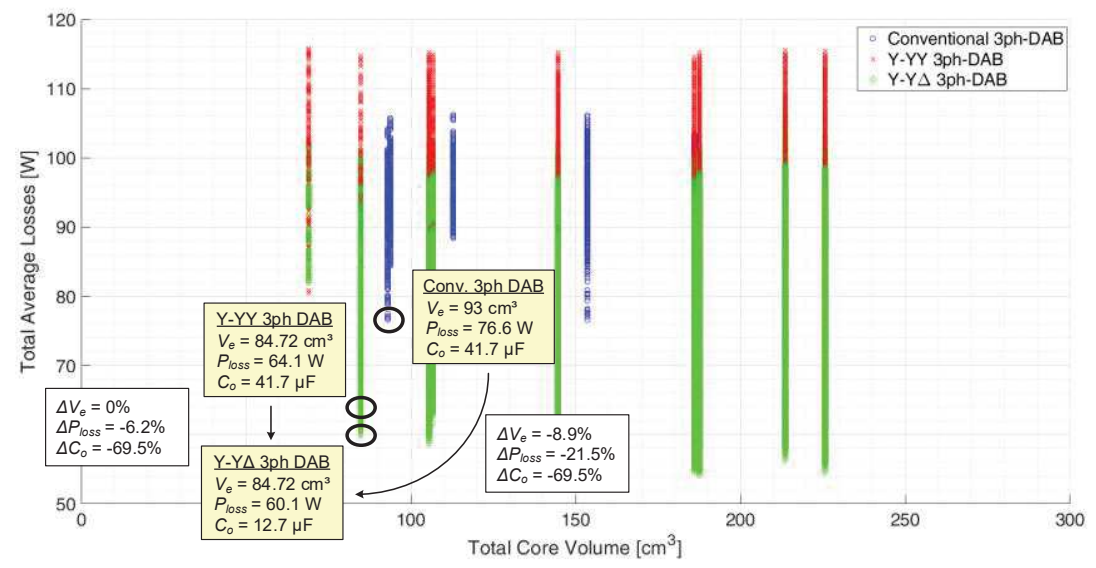

Fig. 7: Selection of the best configuration results regarding total losses, total magnetic core volume and output capacitance. Marked are the chosen designs

\section{Constructed prototypes}

In order to verify the functionality of the circuits, a total of three $4 \mathrm{~kW} 3$ ph-DAB prototypes with two secondary bridges (six independent legs) have been built. The first two prototypes are air-cooled, whereas the final demonstrator is water-cooled. The HV and LV sides are rated from $250 \mathrm{~V}$ to $450 \mathrm{~V}$ and from $36 \mathrm{~V}$ to $52 \mathrm{~V}$, respectively. Moreover, the use of GaN power switches and a maximum volume of 4 liters are required for the final construction.

The following lines describe the chronological path of the executed constructions, from the first prototype, used to check the functionality of both power and control circuits, until reaching the final demonstrator, fulfilling the requirement of maximum volume (see Fig. 8).

The first prototype (Fig. 8a), aimed to verify the basic functionality of both power and control circuits of the also proposed Y-YY 3ph-DAB, uses six Rohm 650 V SiC MOSFETs SCT3030AL, located below the main PCB and screwed onto the heat sink, that are driven with six Analog Devices drivers ADuM4121CRIZ. Twelve Infineon 80 V Si MOSFETs IAUT300N08S5N012 and six TI drivers LM25101A form the low side bridges. The switches are mounted on the PCB top, thermally connected to the heat sink through hundreds of filled \& capped vias positioned under the packages. They are isolated using a flexible thermal interface material between PCB and heat sink and lightly pressed using a hold-down device made of aluminium. The six transformers are built with N97 PQ 50/40 cores, placed on an acrylic support directly above the main PCB.

The second prototype (Fig. 8b), introduces for the first time not only the Y-Y $\Delta 3$ ph-DAB, but also the use of GaN power switches. Moreover, the constructed EMI filters were added to the circuit. The second prototype serves as an air-cooled preliminary version for the final water-cooled construction. Two large air-cooled heatsinks are chosen in order to compare the performance of four different power circuit boards. Two of them comprise the power circuit of the high voltage side, whereas the other two, the one of the low voltage side. The transformers are located between the heatsinks. Both PCBAs of the HV side utilise six GaN Systems $650 \mathrm{~V}$ top-side cooled GS66516T GaN HEMTs mounted on the bottom of the PCB. The difference between both HV PCBAs lies in the gate drivers' design. In one PCBA, the power switches are driven with three TI drivers UCC21520ADW, providing isolated bipolar supplies for high- and low-side switches. In the other setup, six Analog Devices drivers ADuM4121ARIZ-1 with active Miller clamping are adopted, providing a unipolar supply and using bootstrap diodes to supply the high side switches.

In turn, one of the LV PCBAs consists of twelve Infineon $100 \mathrm{~V}$ IAUT300N10S5N015 Si MOSFETs and six TI drivers UCC27212A-Q1 with unipolar supplies. The high side drivers are operated through internal bootstrap diodes. The cooling method is similar to the one described for the LV Si MOSFETs 
in the first prototype. GaN Systems $100 \mathrm{~V}$ GS61008T GaN HEMTs are adopted for benchmarking in the other LV PCBA. The power switches are driven with a unipolar supply through six TI drivers LM5113-Q1 with internal bootstrap diodes. The cooling method is similar to the one described for the HV GaN switches.

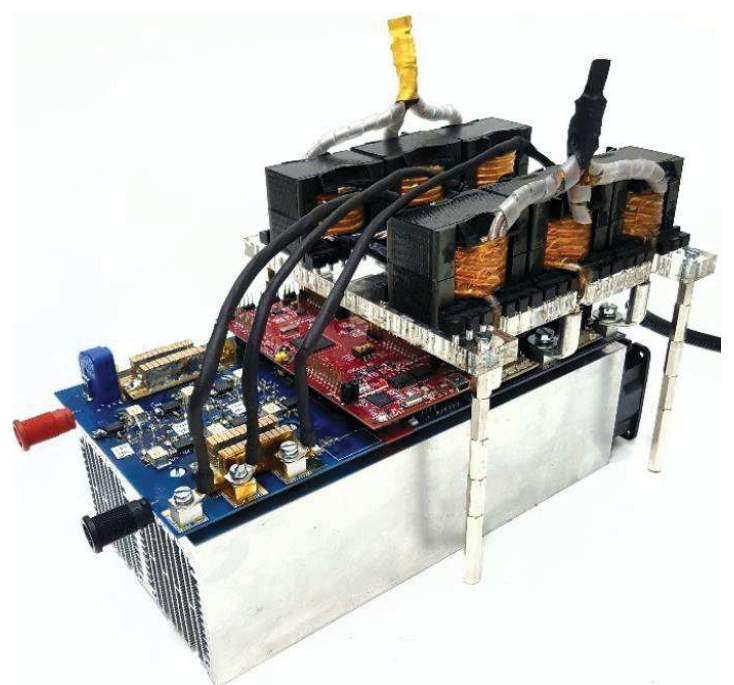

a)

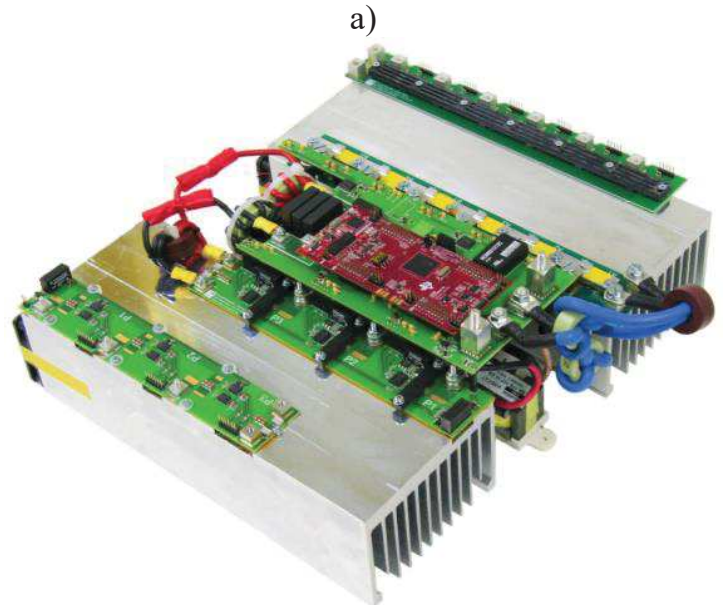

b)

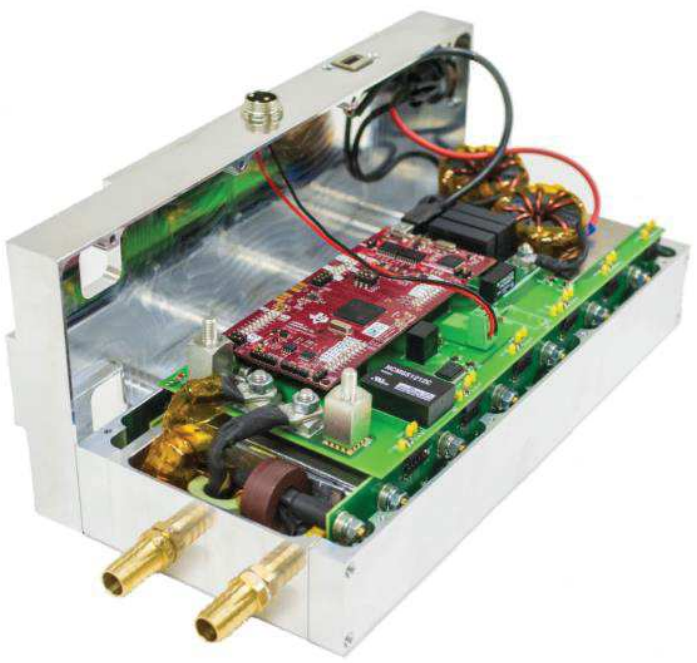

c)

Fig. 8: Chronological evolution of the executed constructions: a) first, b) second and c) third prototypes
The hardware of the third prototype is very similar to the one of the second construction. The main differences between both constructions are the introduction of a water cooled heatsink and the "3D" arrangement of PCBs and transformers, as shown in Fig. 8 c). The six transformers are built with N97 PQ 40/30 cores and are encapsulated inside a cavity in the watercooled heat sink, directly below the controller PCB. The complete enclosed casing serves as an electromagnetic shield. The litz wire conductors are terminated using self-developed wire-toboard connectors, which are screwed to SMT bolts from the PCB backside through cut-outs in the heat sink, as can be seen in Fig. 6a. The reduction in the leg length has a direct effect on the value of the leakage inductance. This value has been further reduced from $9.1 \mu \mathrm{H}$ (second prototype) to $7.8 \mu \mathrm{H}$ on average, getting even further away from the specified value of $10.8 \mu \mathrm{H}$ and, consequently, from the calculated optimal design. Finally, the second and third prototypes use the same control board. It is composed by, among other things, input and output power connectors, measurement circuitry, auxiliary power supplies, digital isolators and a piggyback TI C2000 ${ }^{\text {TM }}$ LAUNCHXL-F28379D

LaunchPad $^{\mathrm{TM}}$. This LaunchPad development kit uses a dual core 32-bit MCU TMS320F28379D, which in turn is responsible for the control loop, PWM generation and communication. The final demonstrator has a volume of $2.9 \mathrm{~L}$ and weighs $4.5 \mathrm{~kg}$. Further measures to reduce both weight and volume are already identified.

As already mentioned, due to geometric constrains, the delivered transformers did by far not reach the desired leakage inductance. The obtained performance regarding power losses is far away from the designed one. The calculations indicate an increase concerning the overall average losses of approximately $19.8 \%$, as shown in Fig. 9a. In turn, Fig. 9b illustrates the deviation of the operating area from the constructed second prototype and the specified system at nominal power. 


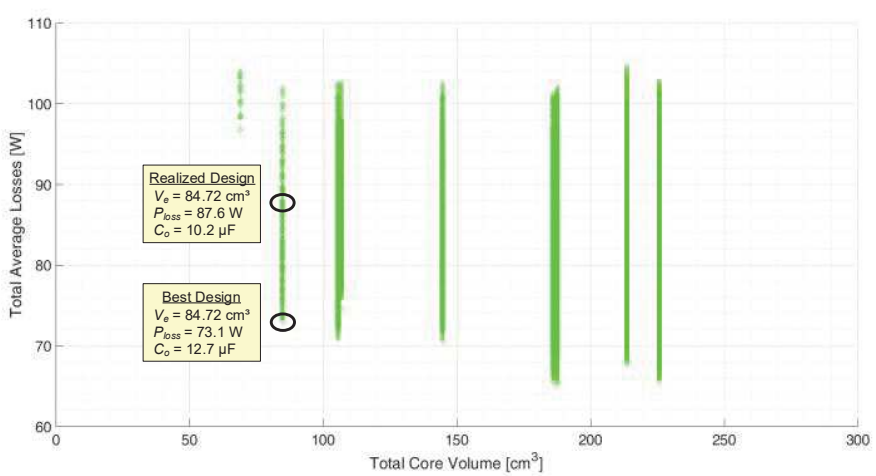

a)

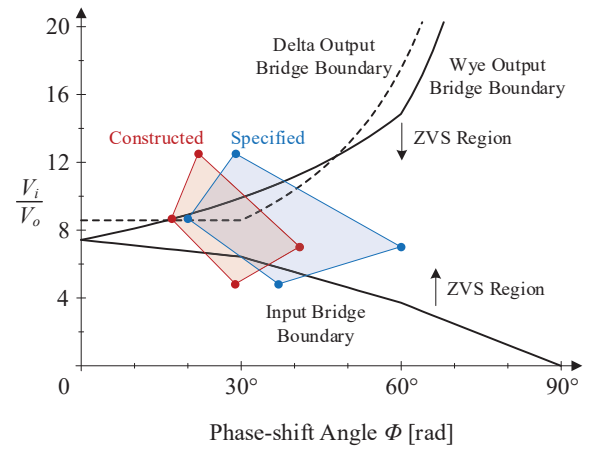

b)

Fig. 9: a) Selection of the best configuration results for the Y-Y $\Delta 3$ ph-DAB using GaN switches. Marked are the best configuration and the realized construction and b) divergent operating areas at nominal power of the specified system and the constructed second prototype

\section{Experimental results}

The oscilloscope waveforms in Fig. 10 present the current through the transformer coils and voltage across the power switches over the time during operation in steady state for $P_{o}=4 \mathrm{~kW}, V_{i}=400 \mathrm{~V}$ and $V_{o}=48 \mathrm{~V}$. In addition, a curve obtained by simulation under identical condition is also provided to verify the correct functionality of the circuit. The HV drain-to-source voltage across the switch $s_{1}$ $\left(U_{d s_{-} S_{1}}\right)$ is represented by the magenta waveform and the $\mathrm{LV}$ drain-to-source voltage across $s_{7}$ $\left(U_{d s_{-} S 7}\right)$ by the yellow one. The transformer primary side current $i_{L a_{-} p}$ is represented by the green waveform and the secondary side $i_{L a_{-} s}$ by the blue one. The vertical scales of each channel are given in the picture and the time scale is $2 \mu \mathrm{s} /$ div. The current through the primary side power switch $s_{1}$ can be extracted directly from $i_{L a_{-} s}$ during the on-time, when $U_{d s_{-} S 1}$ is zero. On the other hand, the current through $s_{7}$ is the inverse of $i_{L a_{-} S}$ during the on-time. These currents are represented through dashed lines. As the currents are completely symmetrical, the waveforms of the other switches are identical except for their phase delays. The juxtaposition of the experimental and simulation results clearly shows that the constructed circuit behaves as expected.

Furthermore, numerous efficiency curves versus transferred power, taken from the third prototype working in direct and reverse power flow directions by keeping the high voltage level constant at $400 \mathrm{~V}$ and varying the low voltage side from $36 \mathrm{~V}$ to $52 \mathrm{~V}$ in five steps, are presented in Fig. 11. A conservative dead time of $200 \mathrm{~ns}$ is adopted as a safety precaution against damage in the initial testing phase. There is an efficiency penalty due to increased diode's forward / HEMT's reverse voltage drop, but it can be minimized by optimizing the system dead time [12]. The obtained efficiency curves are very similar, while the values for direct power transfer are slightly higher than for the reverse operation. For nominal power, all curves are above $94 \%$, reaching a peak of approximately $97 \%$.

Tests are performed under the worst-case voltage combinations at nominal power with ambient temperature of $25^{\circ} \mathrm{C}$ and cooling fluid temperature of $20^{\circ} \mathrm{C}$. Thermal images (Fig. 12) show the HV and LV power switches and the whole system in thermal stationary state. The temperatures of all components are considerably higher than the ambient temperature, however in an acceptable range since the system has to work under higher ambient temperatures of up to $85^{\circ} \mathrm{C}$ and with a cooling fluid temperature of up to $55^{\circ} \mathrm{C}$. On the other hand, the temperature increase on the LV DM chokes is very critical. The measured temperature exceeds $92^{\circ} \mathrm{C}$ under operation with output voltage equal to $36 \mathrm{~V}$, an increase of approximately $67^{\circ} \mathrm{C}$ considering an ambient temperature of $25^{\circ} \mathrm{C}$. It is noteworthy that the LV EMI chokes are not water-cooled in this setup. For operation under higher ambient temperatures, it is necessary to improve their cooling, e.g. through encapsulation. Temperatures on the encapsulated transformers are also observed through five type $J$ thermocouples located between two cores, approximately at the height of their gaps. With the temperature of the 
cooling medium regulated at $20^{\circ} \mathrm{C}$, a maximum temperature variation of approx. $50 \mathrm{~K}$ is achieved, which is consistent with the value adopted in the design. It is noteworthy that the adopted ferrite material N97 reaches its lowest core losses at approximately $100{ }^{\circ} \mathrm{C}[13]$, which it is expected to reach with the adopted design using a cooling medium temperature value of $55^{\circ} \mathrm{C}$.

A full-order small-signal model has been derived based on phasor transformation technique [14]. Adopting this model, an output current control loop is implemented using a simple digital PI
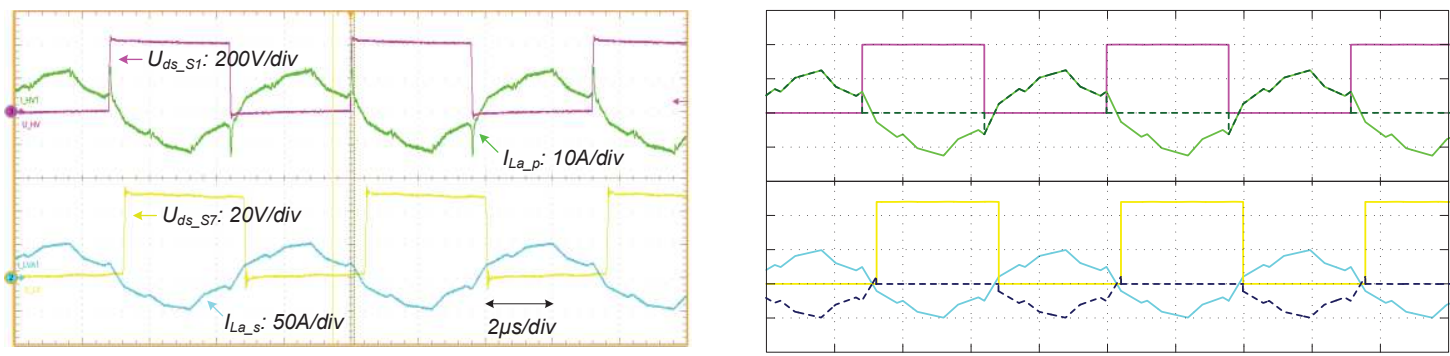

Fig. 10: Juxtaposition of experimental (left) and simulation (right) results for direct power flow direction at rated voltage and power $\left(P_{o}=4 \mathrm{~kW}, V_{i}=400 \mathrm{~V}\right.$ and $\left.V_{o}=48 \mathrm{~V}\right)$
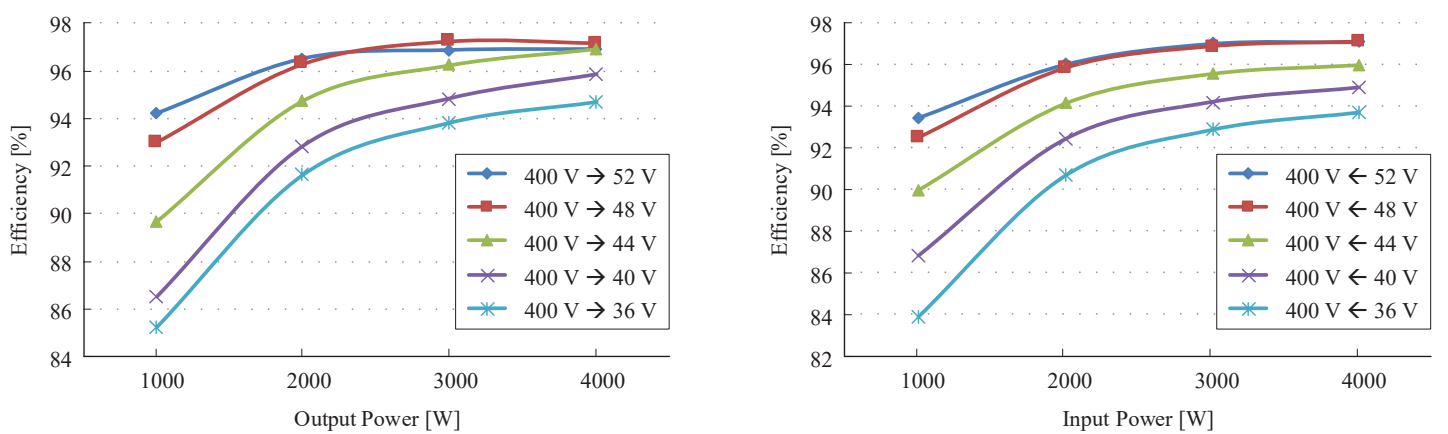

Fig. 11: Efficiency versus power for $V_{i}=400 \mathrm{~V}$ and $V_{o}=36 \ldots 52 \mathrm{~V}$ for direct (left) and reverse (right) power flow directions
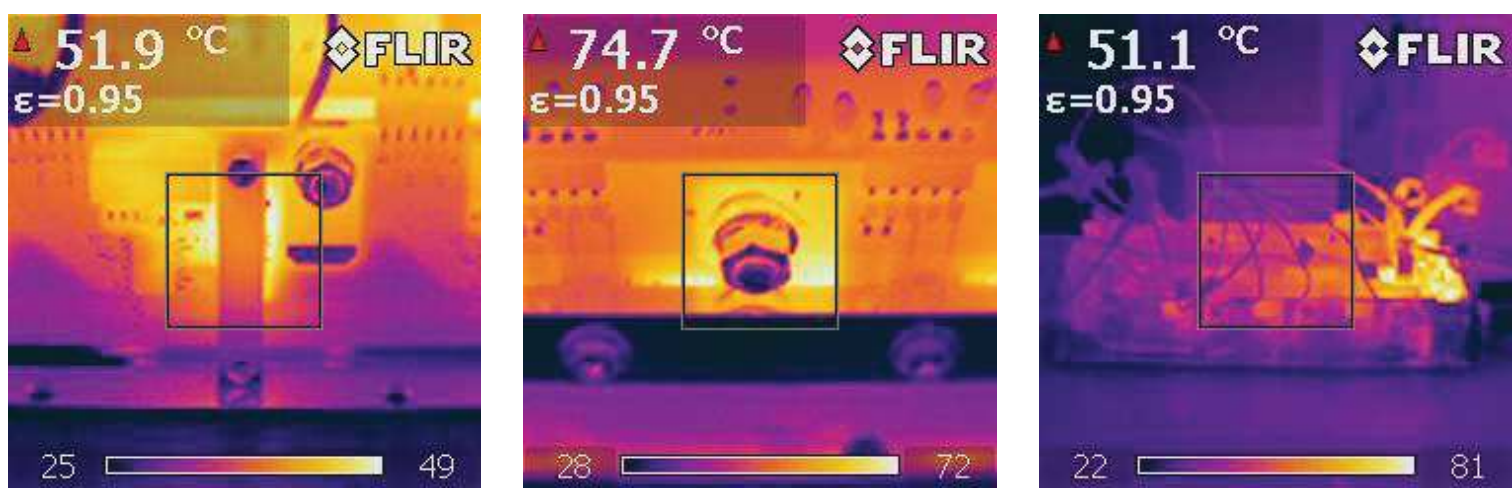

Fig. 12: Worst-case temperatures on the HV GaN switch located on the PCB bottom and measured from the PCB top (left), on the LV switch located under an aluminum hold-down device on the PCB top (middle) and on the complete system viewed from the HV lateral side (right)
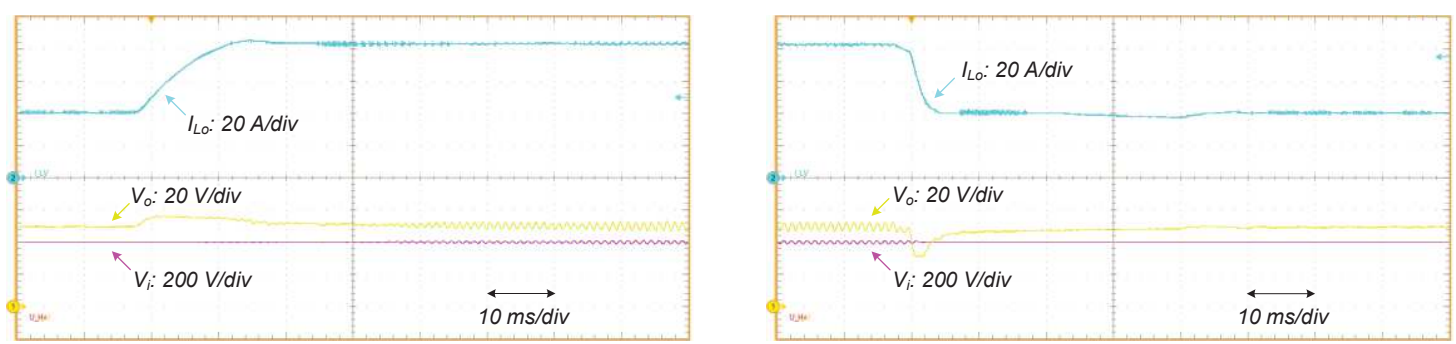

Fig. 13: Output current steps from $42 \mathrm{~A}$ to $84 \mathrm{~A}$ (left) and vice-versa (right) for ( $400 \mathrm{~V} \rightarrow 48 \mathrm{~V}$ ) 
controller. Output current steps from $50 \%$ to $100 \%$ (nominal value) and vice versa performed in direct power flow direction (HV $\rightarrow$ LV) under nominal voltage (400 V, $48 \mathrm{~V}$ ) are shown in Fig. 13. $\mathrm{HV}$ input is represented by the magenta waveform and the controlled DC output current by the blue one. Horizontal and vertical scales are shown individually. The LV output, represented in yellow, is fully controlled by an electronic load. Unfortunately, the used e-load is not able to keep its voltage constant during the step event due to the large time constant of its control loop. If the control loop is realized in a faster way, the circuit can be damaged due to overvoltage.

\section{Conclusion}

This paper introduced a $4 \mathrm{~kW}$ GaN-based isolated bidirectional DC/DC converter intended to interconnect $400 \mathrm{~V}$ HV batteries with $48 \mathrm{~V}$ on-board power supply systems in electrical vehicles. An innovative, highly compact 3D setup is being developed, allowing not only the power switches, but also the magnetic components to be actively water cooled. The heat sink also acts as an EMI shield, protecting the boards against the transformers' magnetic fields. Finally, three $4 \mathrm{~kW}$ prototypes with integrated magnetics were built in order to validate the feasibility of the topology and of the electrical and thermal behaviours of the GaN switches.

\section{References}

[1] E. F. de Oliveira and P. Zacharias, "A modified 12-Pulse Three-Phase Bidirectional Dual Active Bridge DC/DC Converter for E-Vehicles Applications," PCIM Europe 2019, Nuremberg, Germany, 2019, pp. 1-8.

[2] A. Körner, C. Buhlheller, L. Keuck, E. F. de Oliveira. "Spannung im Wandel - Leistungselektronische Wandler für Hybrid- und Elektrofahrzeuge", 7. ETG-Fachtagung, Bad Nauheim, Germany, 2017.

[3] M. G. Txapartegi, "Power electronics for electric \& hybrid vehicles," Yole Développement, Lyon, France, Rep. YD18014, May 2018.

[4] E. F. de Oliveira and P. Zacharias, "Comprehensive Mode Analysis and Optimal Design Methodology of a Bidirectional CLLC Resonant Converter for E-Vehicles Applications," EPE '19 ECCE Europe, Genova, Italy, 2019, pp. P.1-P.10.

[5] D. Rich. (2020). What are the latest updates on dynamically adjustable, dual voltage batteries DC/DC for future electrification?. Innovations in 48V Technology Online. [online]. Available: https://www.automotive-iq.com/events-innovations-in-48v-technology-online/

[6] D. Rich, "Dynamically Adjustable, Dual Voltage Batteries Without a DC/DC for Future Electrification," The Battery Show and Electric \& Hybrid Vehicle Technology Expo 2019, Novi, MI, USA, 2019.

[7] R. W. A. A. De Doncker, D. M. Divan and M. H. Kheraluwala, "A three-phase soft-switched high-powerdensity DC/DC converter for high-power applications”, in IEEE Transactions on Industry Applications, vol. 27, no. 1, pp. 63-73, Jan/Feb 1991

[8] F. B. da Silva, E. F. de Oliveira, T. R. F. Neto and P. Zacharias, "Design Optimization of a Three-Phase Bidirectional Dual Active Bridge DC/DC Converter," PCIM Europe 2018, Nuremberg, Germany, 2018, pp. $1-8$

[9] S. Sprunck, M. Muench and P. Zacharias, "Transient Current Sensors for Wide Band Gap Semiconductor Switching Loss Measurements," PCIM Europe 2019, Nuremberg, Germany, 2019, pp. 1-8.

[10] L. Will, S. Sprunck and P. Zacharias, "Impact of Negative Turn-Off Voltage On Turn-On Losses in GaN EHEMTs," PCIM Europe 2020, Nuremberg, Germany, 2020

[11] GaN Systems, Ontario, Canada. GN002 Application Note - Thermal Design for GaN Systems' Top-side cooled GaNPX®-T packaged devices. Accessed: Jun. 28, 2020. [Online]. Available: https://gansystems. com /wp-content/uploads/2018/10/GN002_Thermal-Design-Guide-for-Top-Side-Cooled-GaNpx-TDevices_Rev-181030.pdf

[12] S. Havanur, "Beware of Zero-Voltage Switching," How2Power Today, 80th issue, Apr. 2016, pp. 1-8. Accessed on Apr. 02, 2020. [Online]. Available: http://www.how2power.com/pdf_view.php?url=/ newsletters/1604/articles/H2PToday1604_design_VishaySiliconix.pdf

[13] TDK Electronics, Munich, Germany. Ferrites and accessories - SIFERRIT material N97 (2017). Accessed: Apr. 22, 2020. [Online]. Available: https://www.tdk-electronics.tdk.com/download/187242/ cf0d9784d3d2438b52ade72fbb86ecf6/pdf-n97.pdf

[14] W. Han and L. Corradini, "Analytical Small-Signal Transfer Functions for Phase Shift Modulated Dual Active Bridge Converters Using Phasor Transformation," ECCE 2018, Portland, OR, 2018, pp. 1442-1448. 